\title{
Clinical and electrophysiological evaluation of dysphagia in myasthenia gravis
}

\author{
Cumhur Ertekin, Nur Yüceyar, İbrahim Aydoğdu
}

\begin{abstract}
Objective-To evaluate dysphagia at the oropharyngeal stage of swallowing and to determine the pathophysiological mechanisms of dysphagia in patients with myasthenia gravis.

Methods-Fifteen patients with myasthenia gravis with dysphagia and 10 patients without dysphagia were investigated by a combined electrophysiological and mechanical method described previously. Laryngeal movements were detected by a piezoelectric transducer and the related submental EMG (SM-EMG) and sometimes the EMG of cricopharyngeal muscle of the upper esophageal sphincter (CPEMG) were recorded during dry or wet swallowing. The results of these electrophysiological variables were compared with those of normal age matched control subjects.
\end{abstract}

Results-In patients with myasthenia gravis with dysphagia, it was found that the time necessary for the larynx to remain in its superior position during swallowing and swallowing variability in successive swallows increased significantly compared with normal subjects and with patients with myasthenia gravis without dysphagia. The total duration of SM-EMG activity was also prolonged in both groups but more severely in the dysphagic patients. Electromyographic activity of the CP sphincter was found to be normal in the dysphagic patients investigated. All the patients with myasthenia gravis with dysphagia had pathological dysphagia limits ( $<20 \mathrm{ml}$ water) whereas other patients except two, were within normal limits.

Conclusions-Because the electrophysiological variables related to oropharyngeal swallowing were prolonged even in patients with myasthenia gravis without dysphagia, it is concluded that the submental and laryngeal elevators are involved subclinically in myasthenia gravis and, because of compensating mechanisms, the patient may not be dysphagic. As the CP-EMG behaviour was found to be normal, a coordination disorder between normal CP sphincter muscle and the affected striated muscles of the laryngeal elevators may be one of the reasons for dysphagia in myasthenia gravis. This method also made it possible to investigate the myasthenic involvement in the laryngeal elevators that cannot be evalu- ated by other electrophysiological methods in myasthenia gravis.

(F Neurol Neurosurg Psychiatry 1998;65:848-856)

Keywords: myasthenia gravis; dysphagia; dysphagia limit; electrophysiology of swallowing; crycopharyngeal muscle; submental EMG

In many patients with myasthenia gravis, fatiguability is usually first seen in the ocular muscles, but as soon as the bulbar muscles are involved, dysphagia occurs together with other bulbar muscle involvement. It has been reported $^{1}$ that $30 \%$ of 175 myasthenic patients had oral, pharyngeal, or laryngeal symptoms and $15 \%$ of them had dysphagia. However, in a larger patient population, difficulty in swallowing was reported to be the initial symptom in $6 \%$ of 1487 patients with myasthenia gravis and during the first month after the onset of disease, $15 \%$ of them had bulbar or oculobulbar involvement. ${ }^{2}$

The prominence of dysphagia together with dysarthria and dysphonia may give rise to an erroneous diagnosis of primary bulbar involvement due to some other causes. If patients with bulbar myasthenia do not exhibit ptosis or diplopia which may be commonly associated with pharyngeal involvement, correct diagnosis can be difficult, and invasive laryngoscopical and fluoroscopical studies are sometimes made by ear, nose, and throat specialists or gastroenterologists in patients with pure bulbar symptoms or in patients with sole manifestations of dysphagia. $^{13-5}$

The mechanism and consequences of dysphagia in myasthenia gravis have been investigated by radiological methods. ${ }^{36-8}$ Manometric studies have been also performed. ${ }^{89}$ In this study, the pathophysiology of dysphagia was investigated by electrophysiological methods described previously. ${ }^{10-12}$

\section{Patients and methods}

Twenty five patients with myasthenia gravis were studied. Their clinical diagnoses were supported by EMG, repetitive nerve stimulation (decrement test), and single fibre EMG (jitter). Clinical responsiveness to anticholinesterase treatment was also important to support the diagnosis of the disorder. Acetylcholine receptor antibodies have not been measured.

Mean age of patients was 44.2 (SD 13.7, range 25-70 years). Fourteen were women. Clinical features of all patients are listed in table 1. The term "dysphagia" was applied when there were abnormal insistent symptoms (nasal regurgitation, the frequent feeling of 
Table 1 Clinical features of patients with myasthenia gravis

\begin{tabular}{|c|c|c|c|c|c|}
\hline Patient & Age/ sex & Duration of $M G(y)$ & $\begin{array}{l}\text { Duration of dysphagia } \\
\text { (months) }\end{array}$ & Initial symptomatology & Present symptomatology ${ }^{\star}$ \\
\hline 1 & $40 / \mathrm{F}$ & 1 & 3 & Ocular & Ocular+bulbar \\
\hline 2 & $46 / \mathrm{M}$ & $5 / 12$ & 5 & Bulbar & Bulbar \\
\hline 3 & $36 / \mathrm{M}$ & $2 / 12$ & 2 & Ocular+bulbar & Ocular+bulbar \\
\hline 4 & $70 / \mathrm{F}$ & 1 & 24 & Generalised & Generalised \\
\hline 5 & $34 / \mathrm{F}$ & 15 & 0.5 & Ocular+bulbar & Generalised \\
\hline 6 & $53 / \mathrm{M}$ & $6 / 12$ & 6 & Ocular+bulbar & Ocular+bulbar \\
\hline 7 & $55 / \mathrm{M}$ & 1 & 6 & Ocular+bulbar & Ocular+bulbar \\
\hline 8 & $52 / \mathrm{M}$ & 5 & 1 & Ocular+bulbar & generalised \\
\hline 9 & $76 / \mathrm{M}$ & 3 & 2 & Ocular & Ocular+bulbar \\
\hline 10 & $32 / \mathrm{F}$ & 4 & 3 & Generalised & Generalised \\
\hline 11 & $48 / \mathrm{F}$ & 1 & 0.5 & Generalised & Generalised \\
\hline 12 & $28 / \mathrm{F}$ & 1 & 2 & Bulbar & Bulbar \\
\hline 13 & $53 / \mathrm{M}$ & 9 & 12 & Generalised & Generalised \\
\hline 14 & $30 / \mathrm{F}$ & 10 & 3 & Ocular & Generalised \\
\hline 15 & $34 / \mathrm{F}$ & 12 & 24 & Bulbar & Bulbar \\
\hline 16 & $50 / \mathrm{M}$ & $3 / 12$ & None & Ocular & Ocular+bulbar \\
\hline 17 & $27 / \mathrm{F}$ & 5 & None & Generalised & Generalised \\
\hline 18 & $69 / \mathrm{M}$ & 1.5 & None & ocular & Generalised \\
\hline 19 & $32 / \mathrm{F}$ & 3 & None & Generalised & Generalised \\
\hline 20 & $25 / \mathrm{F}$ & 1 & None & Ocular & Generalised \\
\hline 21 & $49 / \mathrm{F}$ & 1 & None & Ocular & Ocular+bulbar \\
\hline 22 & $42 / \mathrm{F}$ & 12 & None & Ocular+bulbar & Ocular+bulbar \\
\hline 23 & $39 / \mathrm{M}$ & 2 & None & Generalised & Generalised \\
\hline 24 & $47 / \mathrm{M}$ & 8 & None & Ocular & Generalised \\
\hline 25 & $39 / \mathrm{M}$ & $7 / 12$ & None & Generalised & Generalised \\
\hline
\end{tabular}

${ }^{\star}$ Clinical features of patients with myasthenia gravis; either dysphagic or not at the time of the investigation; none=without dysphagia.

cleaning up of the mouth, the feeling of something being stuck behind the sternum) obtained from their questionaire and abnormal signs (cough during swallowing, accumulation of saliva in the mouth, wet voice after swallowing, palatal paresis) closely related with swallowing disorder. The patients with only uncertain symptoms but lacking any objective signs that could be related to swallowing were not considered to be dysphagic. Therefore, patients with myasthenia gravis were divided into two groups according to presentation of dysphagia at the time of investigation:

(1) Dysphagia was either a prominent complaint (patients $1-5$ in table 1) or had been present until anticholinesterase treatment before they were investigated in this study (patients 6-15 in table 1). These patients were found to be mildly dysphagic on clinical evaluation.

(2) Patients in the second group did not have abnormal signs and symptoms related to dysphagia either at the time of investigation or

Table 2 The results of the questionnaire and clinical findings of patients with myasthesia gravis with dysphagia

\begin{tabular}{|c|c|c|}
\hline & & $\begin{array}{l}\% \\
(n=15)\end{array}$ \\
\hline \multirow[t]{7}{*}{ (A) } & Complaints: & \\
\hline & Cough during swallowing & 100 \\
\hline & Feeling of being stuck behind the sternum & 90 \\
\hline & Nasal regurgitation & 86 \\
\hline & Feeling of the clean up of mouth & 72 \\
\hline & Difficulties in bolus formation and control & 63 \\
\hline & Abnormal posture during swallowing & 54 \\
\hline \multirow[t]{14}{*}{ (B) } & Clinical findings: & \\
\hline & Dysphagia & 100 \\
\hline & Dysphonia & 86 \\
\hline & Facial weakness & 80 \\
\hline & Cough during a swallow & 66 \\
\hline & Saliva accumulation in mouth & 60 \\
\hline & Weak in voluntary cough & 60 \\
\hline & Palatal paresis & 53 \\
\hline & Loss of weight & 46 \\
\hline & Tongue weakness & 40 \\
\hline & Slow laryngeal elevation & 40 \\
\hline & Reduced gag reflex & 26 \\
\hline & Weakness in chewing muscles & 20 \\
\hline & Lung infection & 6 \\
\hline
\end{tabular}

earlier, but other cranial muscles were involved to varying extents (patients $16-25$ in table 1).

Six patients with myasthenia gravis were followed up longitudinally for clinical and electrophysiological evaluations between 1 and 3 months. Table 2 shows the results of the dysphagia questionnaire and the clinical findings closely related to dysphagia in the 15 patients with myasthenia gravis and dysphagia.

Informed consent was obtained from each patient. The investigation was approved by the ethics committee of our University Hospital.

The mean values of swallowing variables obtained from patients with myasthenia gravis were compared with the mean values of 17 (four women, 13 men) normal control subjects (mean age 50.2 (SD 4.2 , range: $40-59$ years)). Individual values obtained from each patient were also compared with the upper limit of the mean value (mean +2 SD) of 75 (34 women, 41 men) normal controls from our laboratory, matched with age. ${ }^{12}$

The electrophysiological methods for evaluating oropharyngeal swallowing have been described previously. ${ }^{10-14}$ Briefly, a mechanical sensor, which consists of a simple piezoelectric wafer with a rubber bulge affixed at its centre, was placed between the cricoid and thyroid cartilages at the midline. Thus upward and downward laryngeal movements associated with swallowing could be detected. The sensor output was connected to one channel of the EMG apparatus and band pass filtered (between $0.01 \mathrm{~Hz}$ and $20 \mathrm{~Hz}$ ). The sensor gave two deflections of generally opposing polarity during each swallow. The first deflection of the laryngeal sensor represents the upward movement of the larynx and the second deflection, its downward movement. The upward and downward deflections of the laryngeal sensor were sometimes diphasic or triphasic for technical reasons. Their shortest time with high amplitude at the beginning of deflexion from the baseline was important and accepted as the 
point of onset. The leading or the trailing edge of the first deflection was used to trigger the delay line circuitry of the recording apparatus so that all signals were time locked to the same instant. The onsets of two deflections in the laryngeal sensor signal recordings are denoted as 0 and 2 . The $0-2$ time interval is a time variable showing the duration of laryngeal superior movement plus relocation during swallowing. ${ }^{10}$ The EMG activity was recorded on a Medelec Model MS-20 EMG apparatus using bipolar silver chloride EEG electrodes taped under the chin over the mylohyoid-geniohyoid anterior digastric muscle complex. The EMG signal was also band pass filtered (between $100 \mathrm{~Hz}$ and $10 \mathrm{~Hz}$ ), amplified, rectified, and then integrated. The surface muscle activity was named submental EMG activity (SM-EMG). As these muscles are the laryngeal elevators, their activities were considered to give information about the onset and duration of swallowing denoted as the A-C interval. The preanalysis time was set at $800 \mathrm{~ms}$ and the total sweep time was set at 2 seconds. At least five successive sensor and EMG signals were recorded for each type of swallowing, dry or wet ( $3 \mathrm{ml}$ tap water). The signals were examined as single traces and as a superimposition and averaging of several.

Cricopharyngeal (CP) muscle of the upper oesophageal sphincter was recorded electromyographically in six patients with myasthenia gravis. The EMG was recorded using disposable concentric needle electrodes. The needle electrode was inserted through the skin at the level of the cricoid cartilage, about $1.5 \mathrm{~cm}$ lateral to its palpable lateral border in the posteromedial direction. High frequency, tonic EMG activity appeared on the oscilloscope screen as the electrode penetrated the muscle. CP muscle activity was also rectified, integrated, and averaged during all types of swallowing.

Besides recording dry and wet swallowing patterns of SM-EMG and the sensor signals reflecting laryngeal movements, piecemeal deglutition and dysphagia limits were also measured electrophysiologically. This test is based on determining piecemeal deglutition which is a physiological phenomenon that occurs when a bolus of large volume is divided into two or more parts which are then swallowed successively. ${ }^{5}$ All patients were instructed to swallow doses of water gradually increasing in quantity from $1 \mathrm{ml}$ to $20 \mathrm{ml}$, and any recurrence of the EMG and laryngeal sensor signals related to swallowing within 8 seconds was accepted as a sign of dysphagia limit. ${ }^{11}$

Mean (SD) values were calculated for all quantities measured and Student's $t$ test was performed to assess the differences in swallowing variables between the groups.

\section{Results}

CLINICAL FINDINGS RELATED TO DYSPHAGIA

In 15 patients with myasthenia gravis and dysphagia, the degree of dysphagia was variable from patient to patient. For instance, it was severe enough for a nasogastric catheter to be used in three patients (1-3). These patients also had diplopia from the onset of the disease, except one (patient 2) in whom pure dysphagia was the first and the only manifestation of myasthenia gravis. Fifteen patients had symptoms and signs closely related to dysphagia and pharyngolaryngeal muscle involvement as shown in table 2 . In all patients with dysphagia,
A
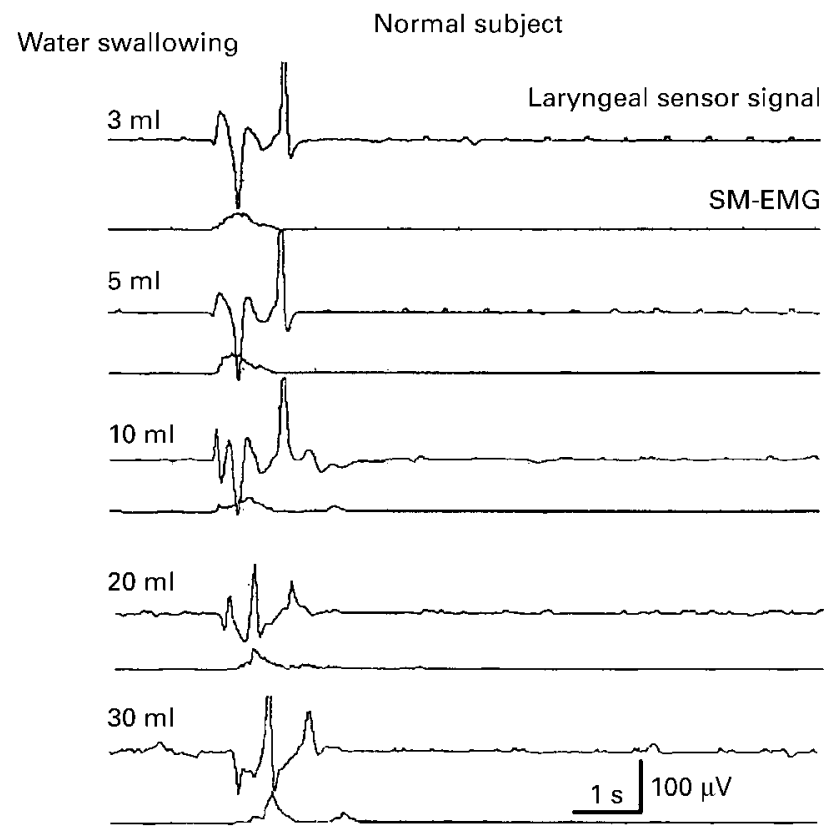

B

Myasthenia gravis with dysphagia

Figure 1 Dysphagia limits of $(A)$ a normal subject and $(B)$ a patient with myasthenia gravis with dysphagia. Note that all volumes, increasing in quantity up to $30 \mathrm{ml}$ water, were swallowed as one bolus in the normal subject whereas the patient with myasthenia gravis began to divide the bolus even at $3 \mathrm{ml}$ water, defining his dysphagia limit. The bolus was divided more as the volume was increased (arrows above the CP-EMG indicate pause during swallowing). Laryngeal sensor signals (top traces in each pair) and integrated EMG activities of the cricopharyngeal muscle of the upper oesophageal sphincter (CP-EMG) (lower traces in each pair in patient with myasthenia gravis) and submental EMG (SM-EMG) (lower traces in each pair in normal subject). 
A

During anticholinesterase treatment
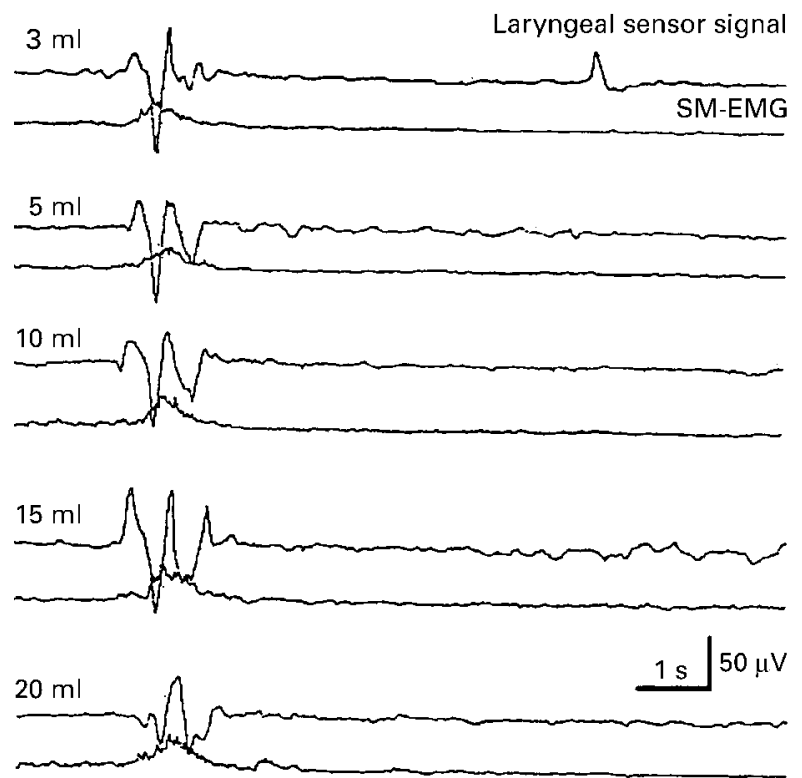

B

Withdrawal of the treatment (After 18 hours)
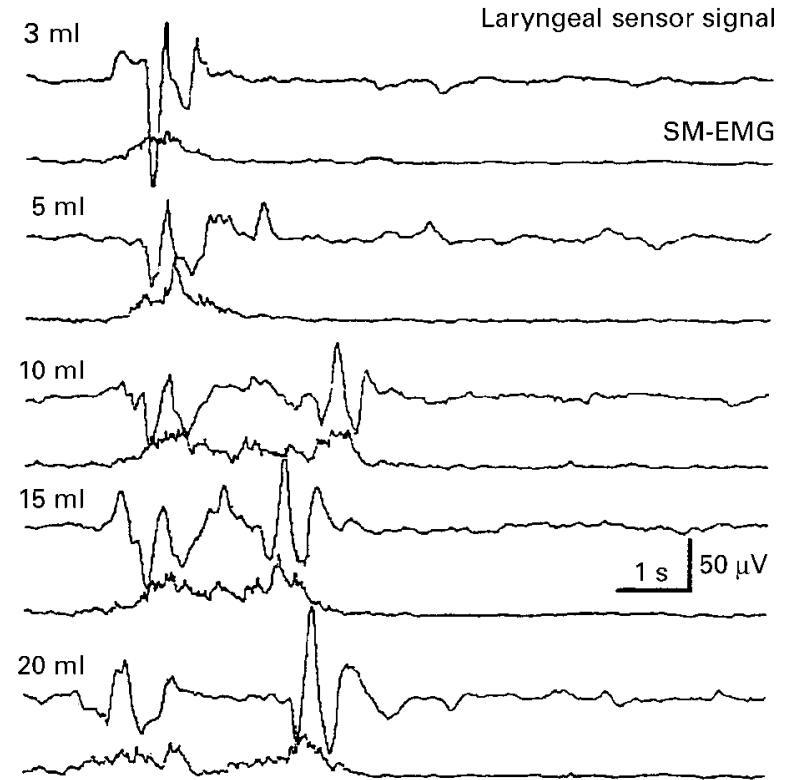

Figure 2 (A) Dysphagia limit of a patient with myasthenia gravis under anticholinestrease treatment was within the normal limits. (B) After the withdrawal of treatment, the dysphagia limit became pathologically low $(10 \mathrm{ml})$ with clinical signs of dysphagia.

anticholinesterase and corticosteroid treatment always produced appreciable recovery from dysphagia. Six of them could be re-examined by electrophysiological methods after effective treatment of myasthenia gravis.

\section{ELECTROPHYSIOLOGICAL FINDINGS RELATED TO} SWALLOWING

(A) Dysphagia limit-The existence of dysphagia in patients with myasthenia gravis could be indicated objectively by this electrophysiological evaluation, showing its usefulness as an electrodiagnostic test. Figure 1 shows dysphagia limits obtained from a patient with dysphagia and from a normal control subject. The normal control subject could swallow up to $30 \mathrm{ml}$ water without dividing the material; but the patient with myasthenia gravis began to divide the bolus while swallowing $3 \mathrm{ml}$ water and he divided it into even more portions as volume was increased. This pattern was never found in normal subjects unless they were required to swallow a volume $>20 \mathrm{ml}$. Therefore the limit of dysphagia was found as $\leqslant 20$ ml. ${ }^{11}$ This method was so sensitive that the dysphagia limit was pathologically low even 18 hours after cessation of anticholinesterase medication in a non-dysphagic patient under anticholinesterase treatment (fig 2). This electrodiagnostic test was performed on patients with myasthenia gravis with dysphagia and the dysphagia limit was found to be $<20 \mathrm{ml}$ in all. Advanced dysphagic cases with myasthenia gravis had a dysphagia limit of $1 \mathrm{ml}$ and sometimes they coughed, suggesting subglottal aspiration. Swallowing recovered both clinically and electrophysiologically after treatment and the dysphagia limit showed complete recovery.

In two of 10 patients without dysphagia, the bolus limit was found to be $20 \mathrm{ml}$, with an occasional divided bolus. The other patients with myasthenia gravis without dysphagia had normal dysphagia limits.

(B) Laryngeal movements and SM-EMGFigure 3 shows a comparison between a normal subject and a patient with myasthenia gravis with dysphagia. One of the important variables was the " $0-2$ time interval", and it was indeed prolonged in patients with myasthenia gravis. The second variable is usually measured from the second deflection peak representing the downward movement of the larynx in superimposed records in which the time between the earliest and latest peak values are quantitatively measured and called "swallowing jitter". ${ }^{10}$ Swallowing jitter can be regarded as the variability in the swallowing apparatus under a given swallowing condition. It can be an important measure of the safety of swallowing during swallowing bolus with various volumes..$^{13}$ It has been found that the swallowing jitter is extremely high in patients with myasthenia gravis with dysphagia compared with normal subjects. Table 3 gives a summary of the statistical results of two groups of patients with myasthenia gravis compared with age matched normal control subjects. The 0-2 interval shows that the upper relocation time of the larynx during dry and wet swallowing was significantly prolonged in patients with myasthenia gravis with dysphagia (table 3 ). It was also prolonged in comparison with the group of patients with myasthenia gravis without dysphagia $(\mathrm{p}<0.05)$. Swallowing jitter in patients with myasthenia gravis with dysphagia lasted longer than that in normal controls and the second group of patients with myasthenia gravis $(\mathrm{p}<0.05)$.

The features of SM-EMG were also pathological in myasthenia gravis. The total duration 
of SM-EMG (denoted as the A-C interval) was significantly prolonged in dry and wet swallowing in both groups of patients with myasthenia gravis compared with normal controls as shown in figure 3. Prolongation of SM-EMG was more severe in patients with dysphagia than in those without dysphagia $(p<0.01)$. The amplitude of the SM-EMG was significantly lower in the dysphagic groups but there was no significant reduction in amplitude in the second group of patients with myasthenia gravis without dysphagia compared with normal control subjects.

Individual values obtained from each patient were also compared with the upper limit of normal values (mean+2 SD). At least one or

Normal subjects
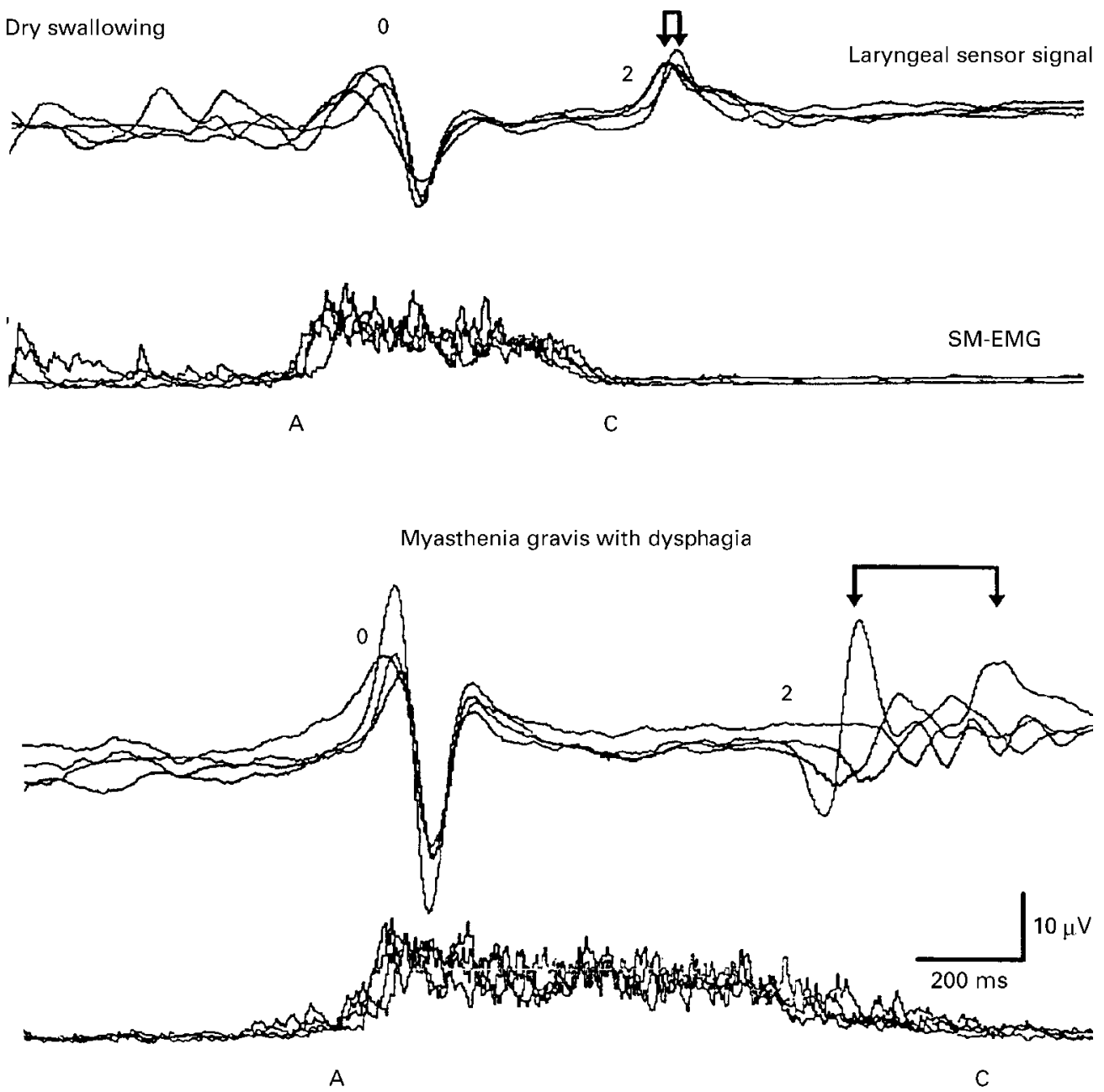

Figure 3 A patient with myasthenia gravis and dysphagia (lower two traces) compared with a normal subject (upper two traces) during dry swallowing. Note that the 0-2 time interval; showing the time for laryngeal upward relocation, swallowing jitter, and the duration of the EMG activity of submental muscle ( $A$-C interval) were prolonged when compared with the normal subject. The arrows above the laryngeal sensor records denotes the swallowing jitter.

Table 3 Summary of the statistical results of patients with myasthenia gravis compared with normal subjects *

\begin{tabular}{|c|c|c|c|c|c|c|c|c|}
\hline \multirow[b]{2}{*}{$(t)$} & \multicolumn{2}{|c|}{ Myasthenia gravis (total) $(n=25)$} & \multicolumn{2}{|c|}{$\begin{array}{l}\text { Myasthenia gravis (with } \\
\text { dysphagia) }(n=15)\end{array}$} & \multicolumn{2}{|c|}{$\begin{array}{l}\text { Myasthenia gravis(without } \\
\text { dysphagia) }(n=10)\end{array}$} & \multicolumn{2}{|c|}{ Normal subjects $(n=17)$} \\
\hline & $3 \mathrm{ml}$ water & Dry swallowing & $3 \mathrm{ml}$ water & Dry swallowing & $3 \mathrm{ml}$ water & Dry swallowing & $3 \mathrm{ml}$ water & $\begin{array}{l}\text { Dry } \\
\text { swallowing }\end{array}$ \\
\hline $\begin{array}{l}0-2 \text { Interval (laryngeal } \\
\text { relocation time) }\end{array}$ & $\begin{array}{l}713(178) \\
\mathrm{p}<0.01\end{array}$ & $\begin{array}{l}672(193) \\
\mathrm{p}<0.01\end{array}$ & $\begin{array}{l}785(175) \\
p<0.001\end{array}$ & $\begin{array}{l}677(168) \\
p<0.01\end{array}$ & 620 (139) NS & 537 (170) NS & $571(85)$ & $534(108)$ \\
\hline $\begin{array}{l}\text { Swallowing variability } \\
\text { (jitter) }\end{array}$ & $\begin{array}{l}120(81) \\
\mathrm{p}<0.05\end{array}$ & $\begin{array}{l}159(111) \\
\mathrm{p}<0.05\end{array}$ & $\begin{array}{l}147(86) \\
\mathrm{p}<0.05\end{array}$ & $\begin{array}{l}197(110) \\
\mathrm{p}<0.01\end{array}$ & $85(60) \mathrm{NS}$ & $88(71) \mathrm{NS}$ & $82(39)$ & $105(46)$ \\
\hline $\begin{array}{l}\text { A-C interval (SM-EMG } \\
\text { duration) }\end{array}$ & $\begin{array}{l}1136(257) \\
\mathrm{p}<0.001\end{array}$ & $\begin{array}{l}1273(360) \\
\mathrm{p}<0.001\end{array}$ & $\begin{array}{l}1237(288) \\
\mathrm{p}<0.001\end{array}$ & $\begin{array}{l}1420(363) \\
\mathrm{p}<0.001\end{array}$ & $\begin{array}{l}1004(130) \\
\mathrm{p}<0.01\end{array}$ & $\begin{array}{l}1052(227) \\
\mathrm{p}<0.05\end{array}$ & $863(89)$ & $896(153)$ \\
\hline SM-E & $51(34) \mathrm{NS}$ & $56(37) p<0.05$ & $49(28) \mathrm{p}<0.05$ & $48(28) \mathrm{p}<0.01$ & 53 (41) NS & 68 (46) NS & & $82(28)$ \\
\hline A-0 interval & $328(161) \mathrm{NS}$ & $382(227) \mathrm{NS}$ & $323(160) \mathrm{NS}$ & 367 (202) NS & $334(170) \mathrm{NS}$ & 404 (273) NS & $286(125)$ & $312(178)$ \\
\hline
\end{tabular}

* Statistical comparisons between the patient groups have not been included in this table (see text).

tSwallowing variables except SM-EMG amp $(\mu \mathrm{V})$ are in ms and the values are shown as mean (SD).

¥A-O interval denotes the time interval at the onset of SM-EMG (A) and the onset of the laryngeal upward movements (0). This value is closely related to the time until triggering of the swallowing reflex..$^{12}$ 


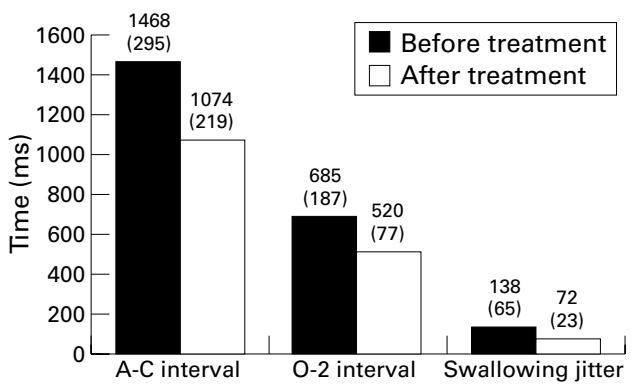

Figure 4 The effect of treatment on the values of $A-C$ interval (SM-EMG activity during swallowing), 0-2 sensor interval (time for laryngeal upward relocation), and swallowing jitter (variability of 0-2 time interval) of six patients with myasthenia gravis. The values are shown as mean (SD).

more electrophysiological abnormalities were encountered in patients with dysphagia. On the other hand, one or more electrophysiological abnormalities were found in six out of 10 patients with myasthenia gravis without dysphagia. Electrophysiological evaluation disclosed that SM-EMG and laryngeal sensor findings are also useful indicators of involvement of laryngeal elevator muscles in patients with myasthenia gravis with and without dysphagia.

After the treatment, all values of SM-EMG and laryngeal movement except amplitude values (0-2 interval, swallowing jitter, and SM-EMG duration) were reduced or shortened toward the normal limits in six patients with myasthenia gravis and dysphagia together with the clinical improvement (fig 4). The reduction in these quantities was found to be significant $(p<0.001$ for A-C interval, $p<0.01$ for swallowing jitter, and $p<0.05$ for $0-2$ interval).

(C) Cricopharyngeal sphincter EMG-CP sphincter EMG was recorded in six patients with myasthenia gravis with dysphagia. The electrophysiological behaviour of CP-EMG during dry or wet swallowing was the same as in normal subjects. ${ }^{10}{ }^{12}$ The CP-EMG behaviour can be seen with the SM-EMG in figure 5 . During a swallow the tonic activity of CP muscle was switched off for about $400 \mathrm{~ms}$ (CP-EMG pause); during this time the SM muscle complex was activated just before the onset of the CP-EMG pause. The normal before and after burst of activity around the
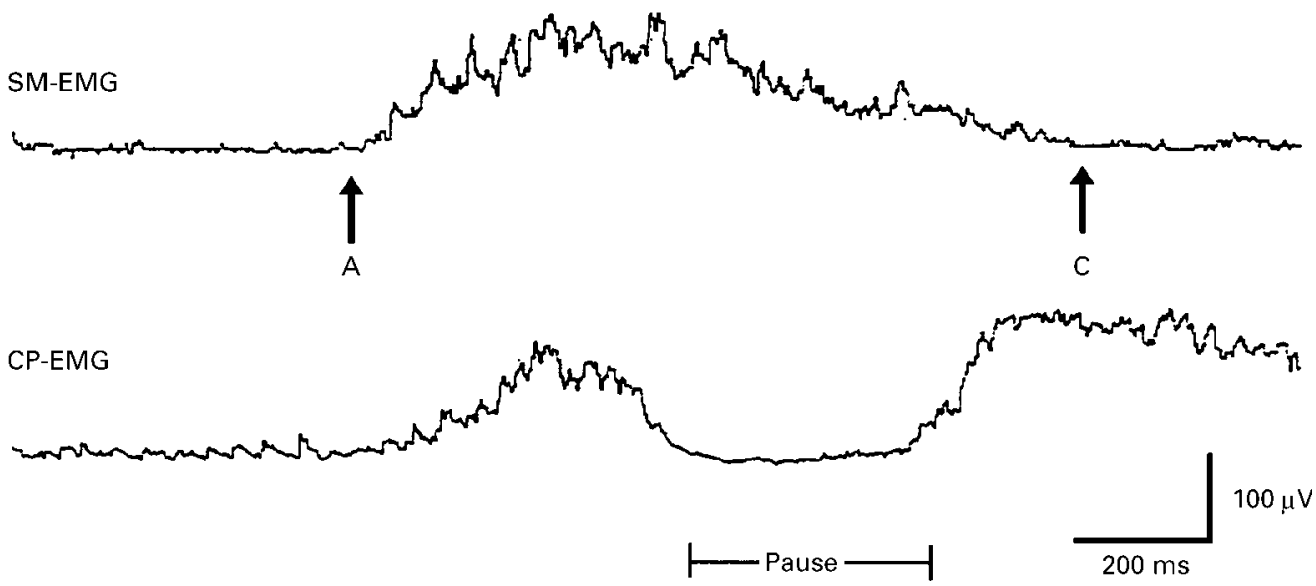

Figure 5 Integrated SM-EMG activity and CP-EMG activity during swallowing of $3 \mathrm{ml}$ water in a patient with myasthenia gravis. Lower pairs are the averaged traces. Upper pairs demonstrate the superposition of all traces. Points $A$ and $C$ denote the onset and the end of submental EMG activity. Pause is the silence of the tonic activity of CP-EMG during swallowing. 


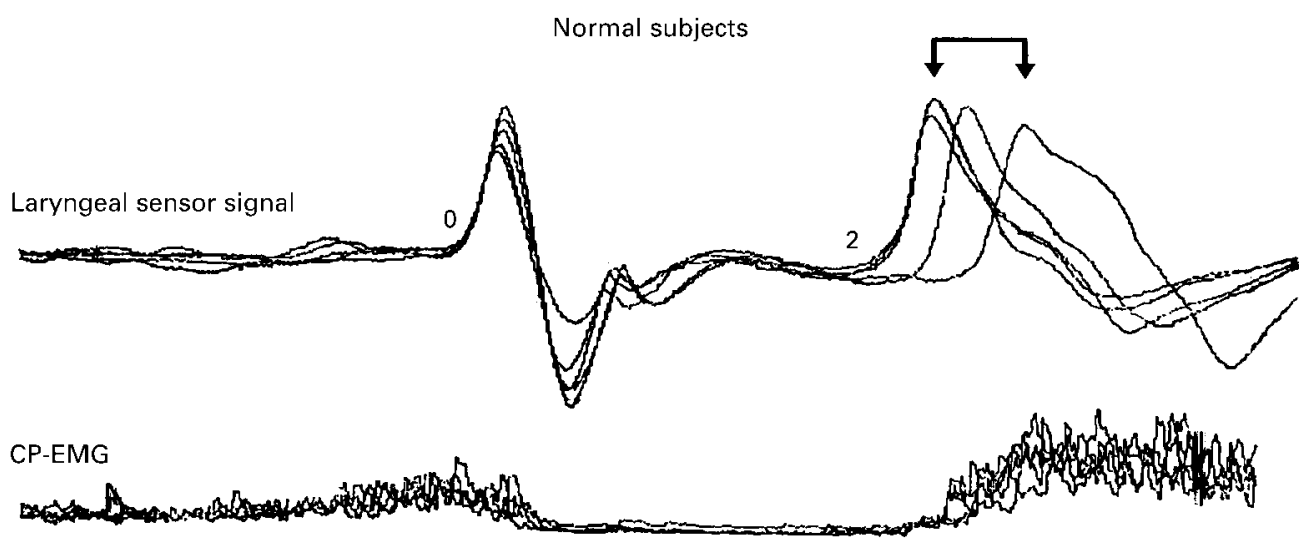

Myasthenia gravis with dysphagia

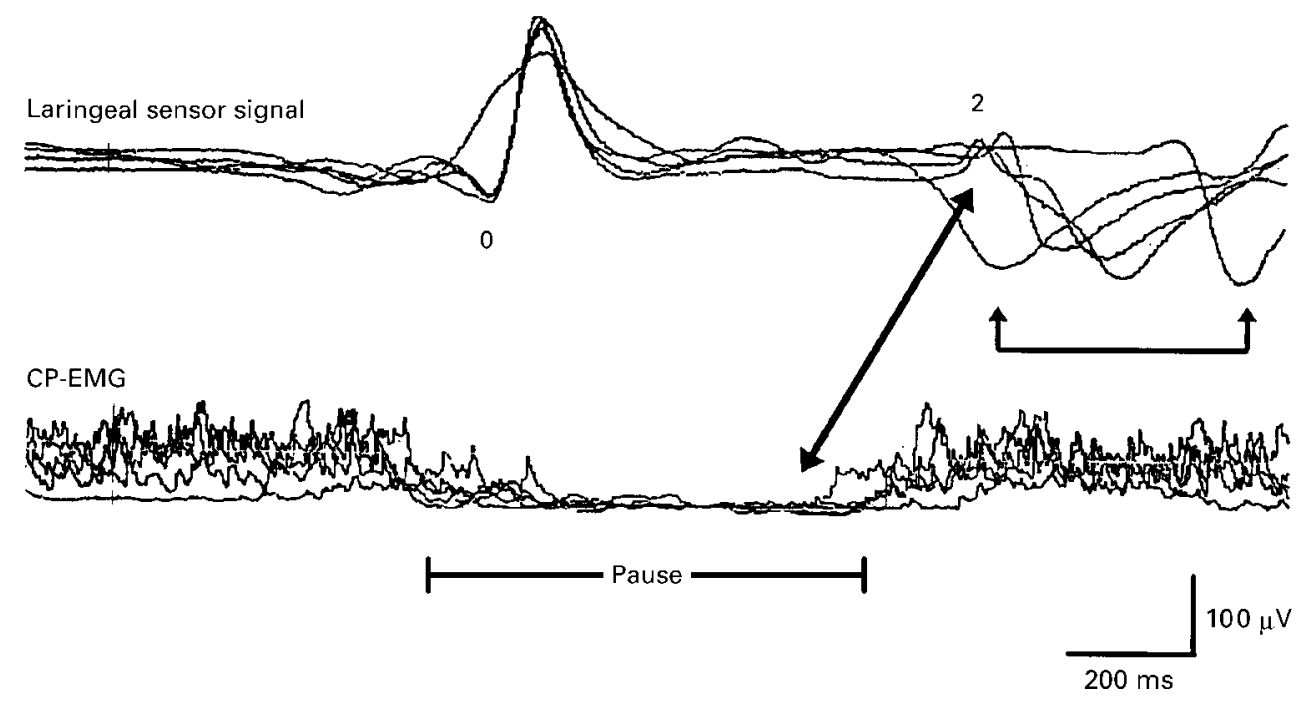

Figure 6 Patient with myasthenia gravis with dysphagia (lower two traces) was compared with the normal subject with regard to the relation between the laryngeal movement and the relaxation of the CP sphincter. Note the prolongation in the laryngeal sensor signal and the increase in swallowing jitter despite the normal CP-EMG pause while swallowing $3 \mathrm{ml}$ of bolus. The CP sphincter is prematurely closed in the patient with myasthenia gravis (oblique arrow). Arrows just below the laryngeal sensor signal in the lower pairs indicate the swallowing jitter with longer values in patients with myasthenia gravis with dysphagia compared with the swallowing jitter (above the laryngeal sensor signal in the upper pairs) of a normal subject.

pause could be easily seen in CP muscle with normal values. ${ }^{10} 12$ Thus no abnormalities were encountered in the CP muscle sphincter in six patients with myasthenia gravis plus dysphagia. On the other hand; coordination of laryngeal movement and the opening or relaxation of the $\mathrm{CP}$ sphincter may be disturbed probably due to the involvement of the suprahyoid and laryngeal elevator muscles and an intact CP sphincter. This is shown in figure 6, where the prolongation of the laryngeal upward movement (0-2 interval) and the increase in swallowing jitter are evident whereas the CP sphincter EMG has its normal pause without compensating the weakness of muscles which elevate the larynx. CP sphincter muscle showed a relatively early closure and probably this differential involvement caused accumulation of pieces of material in the spaces of the pharynx after a swallow and played a part in laryngeal aspiration.

\section{Discussion}

Dysphagia is a common symptom in myasthenia gravis and has been documented in $6 \%$ of 1487 patients with myasthenia gravis as an initial symptom. ${ }^{2}$ If it is the initial symptom and a prominent complaint, ear, nose, and throat specialists or gastroenterologists rather than neurologists may be consulted first. However, such patients are not very common. On the other hand, the incidence of dysphagia is probably underestimated. Based on our findings in this study, we have suggested two reasons for this. Firstly, dysphagia is so mild and insidious that the patient cannot be aware of his or her symptoms. Frequent involvement of ocular muscles apart from the pharyngeal and laryngeal muscles may dominate the complaints of patients with myasthenia gravis, so that dysphagia may be unnoticed by the patient. We have experienced this finding in our patients. Despite the common involvement of ocular 
and pharyngeal muscles, the complaint of diplopia was more obvious and dysphagia has been missed by both the patients and the physicians. On the other hand, the myasthenic involvement in pharyngeal and laryngeal muscle groups has been rapidly cured with early and effective anticholinesterase treatment.

The second reason for missing a swallowing disorder in myasthenia gravis is that the tongue, pharyngeal muscle of the oral floor, and other laryngeal elevators cannot be properly investigated by conventional electrodiagnostic methods. Myasthenic involvement was determined in the laryngeal elevators by means of the electrophysiological methods described. SM muscles have two actions in swallowing; one is the protection of the larynx by elevation, the second is the transportation of the bolus by a secondary support to the tongue to pump. ${ }^{15-17}$ We have found that the SM muscles were activated for an abnormally long period during swallowing and the duration of SM-EMG was much longer in patients with myasthenia gravis with and without dysphagia. The prolongation of laryngeal upward movement and its relocation during swallowing ( $0-2$ time interval) were also strictly related with long duration of SM-EMG. The prolonged activity of the weak SM muscles to keep the larynx at an anterosuperior relocation position during swallowing, must be the cause of longer duration of these variables. As these abnormalities are quite frequent in patients with myasthenia gravis without dysphagia, it can be proposed that SM muscles and other laryngeal elevators are also involved in myasthenia gravis without dysphagia but this can be shown objectively only by electrophysiological variables. Pure electrophysiological involvement of these muscles can be explained on the assumption the muscles are probably weak but are normal clinically. This is similar to the fact that in myasthenia gravis, although skeletal muscle power may be normal clinically it may be abnormal electrodiagnostically. ${ }^{18-20}$ Subclinical weakness of the muscles investigated and weakness of other muscles such as the tongue and pharyngeal constrictor muscles, which are primarily related to swallowing, may not always result in dysphagia. Because either voluntary compensation (when the patient is aware of his or her swallowing impairment), or involuntary compensation mechanisms which occur through adjustments in the swallowing apparatus itself ${ }^{11}$ may take place in the presence of weakness of oropharyngeal muscles. Thus patients with myasthenia gravis with a subclinical swallowing impairment may subconsciously alter the consistency of ingested food and the speed of eating and drinking and there may not be overt symptoms of dysphagia. ${ }^{22}$ The prolongation of SM-EMG duration and 0-2 laryngeal sensor interval might be a type of compensating mechanism to prevent aspiration by keeping the larynx closed and elevated during swallowing. The increase in jitter and the lowering of the dysphagia limits in patients are another type of compensation for swallowing in the case of weakness of oral and pharyngeal muscles.
Swallowing jitter can be adjusted from one swallow to another according to peripheral conditions. This may also be related to the change in the number of muscle fibres in transmission block from one swallow to another even if the same bolus volume is swallowed. This would result in further fluctuations as detected by the second deflections of the laryngeal sensor. After the anticholinesterase treatment, the increase in swallowing jitter has declined towards normal limits accompanied by clinical improvement.

If the bolus is large enough, it is not sufficient to increase the times of swallowing events such as the $0-2$ interval or SM-EMG duration; instead it becomes necessary to divide the bolus into portions and swallow them successively in piecemeal deglutition. Thus in the case of myasthenia gravis with dysphagia this mechanism works and the dysphagia limit is lowered below a $20 \mathrm{ml}$ water bolus, which is the upper limit in normal subjects. ${ }^{11}$ The lowering of the dysphagia limit therefore represents a more advanced stage of dysphagia (the clinically apparent stage). Indeed, whereas all of our patients with myasthenia gravis with dysphagia had abnormal dysphagia limit values, only two patients without dysphagia had a dysphagia limit at a $20 \mathrm{ml}$ water bolus.

EMG activity of the CP sphincter was normal in all patients investigated. The EMG behaviour of this sphincter muscle during swallowing has not been found different from

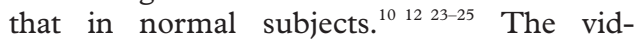
eofluoroscopic and cinefluoroscopic studies have also disclosed that the CP sphincter is almost always normal in myasthenia gravis. ${ }^{3}{ }^{7}$ However, Huang et al showed a significant decrease in sphincter pressure in a manometric study but they found that the relaxation and coordination of this sphincter were within normal limits. Similar to our findings, fluoroscopic studies disclosed that the tongue, laryngeal elevators, and pharyngeal constrictors are mostly involved in myasthenia gravis with dysphagia. ${ }^{3826}$ The selective involvement of the swallowing apparatus in myasthenia gravis can be explained by the fact that CP muscle is more dependent on a central swallowing programme and the central pattern generator in the bulbus, ${ }^{27}$ and its action is semiautomatic and involuntary despite its striated nature. ${ }^{28}$

Although the CP muscle of the upper esophageal sphincter is intact, this finding will be irrelevant as the myasthenic process advances to involve the tongue and the pharyngeal constrictor muscles, which pump and sweep the bolus, and the submental and other laryngeal elevator muscles which protect the larynx during swallowing. A coordination disorder between these suprahyoid laryngeal elevator muscles which are involved in myasthenia gravis and the intact cricopharyngeal muscle of the upper esophageal sphincter may be one of the causes of dysphagia and aspiration in MG. We are grateful to Professors G Celebi and N Celebisoy for their
invaluable help. This study was supported by a grant from the invaluable help. This study was supported by a grant from the
Turkish Scientific and Technological Research Council (TUTưrkish Scientific and Technological
BİTAK) (Project No SBAG-1739). 
1 Carpenter RC, Mc Donald TJ, Howard EM. The otolaryngologic presentation of myasthenia gravis. Laryngoscope

2 Grob D, Arsura EL, Brunner NG, et al. The course of myasthenia gravis and therapies affecting outcome. Ann $N$ Y Acad Sci 1987;505:472-99.

3 Donner MW, Silbiger ML. Cinefluorographic analysis of pharyngeal swallowing in neuromuscular disorders. $A m \mathcal{F}$ Med Sci 1966; 25:600-16.

4 Khan OA, Campbell Ww. Myasthenia gravis presenting as dysphagia: clinical considerations. Am f Gastroenterol 1994 89:1083-5.

5 Logeman JA. Evaluation and treatment of swallowing disorders. Austin, TX: Pro-ed, 1983:76.

6 Felson BJ, Klatte EC. Pharyngeal and upper esophageal dysphagia. FAMA 1976;235:2643-6.

7 Kilman WJ, Goyal RK. Disorder of pharyngeal and upper esophageal sphincter motor function. Arch Intern Med 1976;136:592-601.

8 Kluin KJ, Bromberg MB, Feldman EL, et al. Dysphagia in elderly men with myasthenia gravis. F Neurol Sci 1996;138: $49-52$

9 Huang MH, King KL, Chien KY. Esophageal manometric studies in patients with myasthenia gravis. $\mathcal{F}$ Thorac Cardiovasc Surg 1988;95:281-5.

10 Ertekin C, Pehlivan M, Aydogdu I, et al. An electrophysiological investigation of deglutition in man. Muscle Nerve 1995;18:1177-86.

11 Ertekin C, Aydogdu I, Yüceyar N. Piecemeal deglutition and dysphagia limit in normal subjects and in patients with swallowing disorders. F Neurol Neurosurg Psychiatry 1996; 61:491-6.

12 Ertekin C. Clinical diagnosis and electrodiagnosis of swallowing disorders. In: Swenson MR, ed. Disorders of speech and swallowing. AAEM, 19th annual courses. Minnesota: Johnson, 1996;22-33.

13 Ertekin C, Aydogdu I, Yüceyar N, et al. Effects of bolus volumes on the oropharyngeal swallowing - an electrophysi-
ological study in man. Am 7 Gastroenterol 1997;92:2049-53.

14 Pehlivan M, Yüceyar N, Ertekin C, et al. An electronic device measuring the frequency of spontaneous swallowing: digital phagometer. Dysphagia 1996;11:259-64.
15 Cook IJ. Normal and disordered swallowing: new insights. Baillere's Clinical Gastroenterology 1991;5:245-67.

16 Dodds WJ, Stewart ET, Logeman JA. Physiology and radiology of the normal oral and pharyngeal phases of swallowing. AfR Am f Roentgenol 1990;154:953-63.

17 Mc Connell FM Scerenko D, Jackson RT, et al. Timing of major events of pharyngeal swallowing. Arch Otolaryngol Head Neck Surgery 1988;114:1413-8.

18 Sanders DB, Howard JF. Single-fiber electromyography in myasthenia gravis. Muscle Nerve 1986;9:809-19.

19 Sanders DB, Howard JF, Johns TR. Single-fiber electromyography in myasthenia gravis. Neurology 1979;29:68-76.

20 Stalberg E, Sanders DB. Electrophysiological tests of neuromuscular transmission. In: Stalberg E, Young RR, eds. Clinical neurophysiology. London: Butterworths, 1981: $88-116$.

21 Buchholz DW, Bosma JF, Donner MW. Adaptation, compensation and decompensation of the pharyngeal swallowing. Gastrointest Radiol 1985;10:225-33.

22 Jones B, Ravich WJ, Donner MV, et al. Pharyngoesophageal interrelationships: observations and working concepts. Gastrointest Radiol 1985;10:225-233.

23 Elidan J, Schochina M, Gonen B, et al. Manometry and electromyography of the pharyngeal muscles in patients with dysphagia. Arch Otolaryngol Head Neck Surg 1990;116: 910-3.

24 Shipp T, Deatsch WW, Robertson K. Pharyngoesophageal muscle activity during swallowing in man. Laryngoscope 1970;80:1-16.

25 Van Overbeek JJ, Wit HP, Paping RH, et al. Simultaneous manometry and electromyography in the pharyngoesophageal segment. Laryngoscope 1985;95:582-4.

26 Silbiger ML, Pikielney R, Donner MW. Neuromuscular disorders affecting the pharynx-cineradiographic analysis. Invest Radiol 1967;2:442-8.

27 Miller AJ. Deglutition. Physiol Rev 1982;62:129-84.

28 Bonington A, Mahon M, Whitmore I. A histological and histochemical study of the cricopharyngeus muscle in man. f Anat 1988;156:27-37. 